\title{
The ultradian clock: not to be confused with the cell cycle
}

\section{David Lloyd}

Biochemical reactions that constitute the metabolic network generally have relaxation times in the range of $0.1 \mathrm{~ms}$ to $100 \mathrm{~s}$, and are but one component of the massively interconnected web of the total cellular system ${ }^{1,2}$. For at least 5 decades, this system has been referred to as a temporal hierarchy, but as pointed out by Yates ${ }^{3}$, it is really a heterarchy in which the levels of organization are not separately and clearly defined, but interact strongly at every level and even directly between widely separated levels. The time structure of life $\mathrm{e}^{4}$ is at least as complex as it its spatial structure - but, whereas the spatial structure is documented precisely to $\AA$ resolution for many macromolecules and we have extensive information on subcellular ultrastructure, our appreciation of the temporal organization is less impressive. Details of molecular dynamics and enzyme kinetics have been examined in vitro until recently, but with the development of new noninvasive imaging techniques, they are now increasingly studied in vivo. Rapid progress towards minimizing our ignorance is being made even on a $\mu$ s time-scale resolution ${ }^{5}$.

Despite the clarity and astonishing spatial resolution of medical imaging techniques, the direct non-invasive probing of the whole organism is rather less feasible 6 . The system developed by Kuriyama and his colleagues using a self-synchronized continuous culture of yeast ${ }^{7}$ provides a unique opportunity to extend our understanding of the minuteto-minute development of the organism through multiple cycles in experiments that might be continued for days, weeks or months. The unperturbed progression of state changes can be monitored, as if in a single organism, although the population density is $10^{9}$ yeasts cells per $\mathrm{ml}$. So, there is plenty of both cellular and extracel- lular material for discrete time samples for biochemical analysis. So far, 15 papers have been published, and the essential discoveries recently reviewed ${ }^{8,9}$. In a nutshell, the picture that emerges is one of an exquisitely and intensively interwoven network of temporal controls that encompass every aspect of cellular function, including electron transport, membrane transport, energy transduction, metabolism, biosynthesis transcription, membrane translation and ribosome assembly, organelle biogenesis, membrane flux and trafficking, and nuclear and cell-division cycle progression ${ }^{10}$. Most importantly, the entire system is tied to a metronomic time-base (in yeast, a 40-min interval) that provides a simplifying principle, a temporal ordering to the enormous complexity of the living state. Strong evidence for a similar organization of the time structures of other yeasts and protists has been summarized ${ }^{11}$.

Recently, the clarity of this picture has been undermined ${ }^{12,13}$. Tu et al. ${ }^{12}$, in attempting to further advance our understanding in this area, have unwittingly used markedly different culture conditions (especially with respect to aeration), and in so doing have produced a different system from that of Kuriyama, the one with respiratory oscillations first described in 1978 and extensively researched ever since ${ }^{14}$. The phenomenon they describe $(\tau$ (period) $=3-4 \mathrm{~h}$ ) is the cell-division cycle (mitotic) oscillation, not the shorter-period ultradian clock cycle. Although the cell-division cycle is sometimes referred to as a clock, its duration is temperature and nutrient dependent, and therefore is a rhythm rather than a clock.

The Opinion article by Tu and McKnight ${ }^{13}$ further confuses readers on several issues. Its main hypothesis, that "cyclic changes in a cell's metabolic state might be a fundamental driving force for biological oscillations", and the authors' comparisons of metabolic cycles with circadian cycles, the hibernation cycle and the sleep-wake cycle (all of which remain a 'mystery') is poorly expressed and says nothing new. Their paper fails to cite key advances in the field. The redox-switching mechanism that lies at the core of the cellular network, the ultradian $\operatorname{clock}^{9,10}$, drives nothing as it is one of many central components of the global system.

Microbiology, Main Building, Cardiff School of Biosciences, P.O. Box 915, Cardiff University, CF10 3TL, Wales, UK. e-mail: Iloydd@cf.ac.uk doi:10.1038/nrm 1980-c1

1. Goodwin, B. C. Temporal Organization of Cells (Academic Press, London, 1963).

2. Lloyd, D., Poole, R. K. \& Edwards, S. W. The Cell Division Cycle: Temporal Organization of Cellular Growth and Reproduction (Academic Press, London, 1982).

3. Yates, F. E. Fractal applications in biology: scaling time in biological networks. Methods Enzymol. 210, 636-675 (1992)

4. Aon, M. \& Cortassa, S. Dynamic Biological Organisation: Fundamentals as Applied to Cellular Systems (Chapman \& Hall, London, 1997).

5. Digman, M. A. et al. Fluctuation correlation spectroscopy with laser-scanning microscope: exploiting the hidden time structure. Biophys. J. 89, 1317-1327 (2005).

6. Chance, B., Pye, E. K., Ghosh, A. K. \& Hess B. Biological and Biochemical Oscillators (Academic Press, New York, 1973).

7. Satroutdinov, A. D., Kuriyama, H. \& Kobayashi, H Oscillatory metabolism of Saccharomyces cerevisiae in continuous culture. FEMS Microbiol. Lett. 98, 261-267 (1992).

8. Lloyd, D. \& Murray, D. B. The ultradian clock: metronome for the orchestration of cellular coherence. Trends Biochem. Sci. 30, 373-377 (2005).

9. Lloyd, D. \& Murray, D. B. The temporal architecture of eukaryotic growth. FEBS Letts. 580, 2830-2835 (2006).

10. Klevecz, R. R., Bolen, J. Forrest, G. \& Murray, D. G A genomewide oscillation in transcription gates DNA replication and cell cycle. Proc. Nat Acad. Sci. USA 101, 1200-1205 (2004).

11. Lloyd, D. In Ultradian Rhythms in Life Processes (eds Lloyd, D. \& Rossi, E. R.) 5-22 (Springer-Verlag, London, 1992).

12. Tu, B. P., Kudlicki, A., Rowicka, M. \& McKnight, S. L. Logic of the yeast metabolic cycle: temporal compartmentation of cellular processes. Science 310 1152-1158 (2005)

13. Tu, B. P. \& McKnight, S. L. Metabolic cycles as an underlying basis biological oscillations. Nature Rev. Mol. Cell Biol. 7, 696-701 (2006).

14. Mochan, E. \& Pye, E. K. Respiratory oscillations in adapting yeast cultures. Nature New Biol. 242 177-179 (1973). 